\title{
Revenue Management: A Real Options Approach
}

\author{
C.K. Anderson, University of Western Ontario \\ M. Davison, University of Western Ontario \\ H. Rasmussen, University of Western Ontario
}

Revenue management is the process of actively managing inventory or capacity to maximize revenues. The active management typically occurs through managerial levers such as price, promotion, or availability. We present a novel real options approach to revenue management that is specifically suited to the car rental business. We illustrate the concept with actual car rental data. The model produces minimally acceptable prices and inventory release quantities (number of cars available for rent at a given price) as a function of remaining time and available inventory. The pricing and inventory release recommendations of the developed model confirm earlier empirical analysis that suggested current practices discount too deeply early in the booking cycle.

\section{Introduction}

In this paper we derive a new revenue management procedure for the rental car industry. This procedure is obtained by considering the operator of a car rental business to be the holder of a real option very similar to the swing option used in the energy industry. This analogy allows us to determine, under reasonable and broad assumptions, both the value of such a business and the optimal way to manage it.

Revenue management is a process of managing perishable inventories to maximize the total revenue from these inventories. The concept has its origins in the airline industry where, upon departure, unfilled seats are lost revenue opportunities. Revenue management is not limited to airlines, but suitable for numerous retail and service industries, including advertising, car rentals, cruise ships, and flexible manufacturing (Harris and Peacock [17]).

In recent years a new theory of pricing and operating assets in the face of uncertainty and in the presence of some flexibility in operating strategies has been developed. This is the theory of real options (Dixit and Pindyck [10]; Amram and Kulatilaka [1]). The theory of real options in turn uses the framework 
of modern financial options pricing to frame and solve its problems. This formulation allows these problems to be framed as partial differential equations, normally of diffusive type. For general mathematical references on options pricing see (Merton [24], Hull [18], and Wilmott [33]).

Different types of real options exist, depending on the flexibility possessed by the business operator. For example, consider a project with known start-up costs that may be initiated any time in the next year. Such a project may be modeled as an American call option -the real option holder is allowed to "buy into" the project or exercise his option on any date in the next year. Pricing such an option requires a decision about the optimal way in which to exercise it. The two problems are solved in tandem.

We show that the car rental problem corresponds to a different kind of option. We consider the problem of booking cars for a particular future date. The price at which cars may be reserved fluctuates with both deterministic and random components. Now the "exercise" decision occurs at every instantshould I allow bookings at this price or not? Each exercise decision affects subsequent ones, for every car that gets reserved decreases the number of cars which may be rented in the future. It turns out that this problem is very similar to the problem of pricing the "swing" contracts prevalent in the electricity and gas industries (Keppo [20]) and (Jaillet, Ronn, and Tompaidis [19]).

As an example of a swing option contract consider a typical natural gas supply contract. Under such a contract the buyer takes a certain base supply every week at a preset price. The buyer is also able, at his option, to decide each week whether or not he would like to buy more gas at another preset price. If he does decide to take more gas, he is said to "swing up." The complicating factor here is that the option holder is given only a finite number of opportunities to swing up. Pricing this option requires an exercise strategy which tells the option holder how best to deploy these swing opportunities.

In this paper we show how a car rental operator may be considered as the holder of a swing-like option on car rentals. We are able to solve the resulting set of equations to determine both the operator's optimal rental strategy and the value of the business to the operator.

In Section 2 we provide a detailed description of revenue management in the car rental industry. In Section 3 we derive a partial differential equation model to describe the financial aspects of a car rental business. A numerical procedure for extracting generally applicable model solutions is discussed in Section 4. In Section 5 we present analytic solutions for some special cases of this model. We use the analytic results to validate the model of Section 4. Numerical examples for a general case are presented in Section 6. In the final section we discuss model extensions and future work. 


\section{Revenue Management in Car Rentals}

Revenue management has been a topic of research and practical interest for airlines since the early 1970s. Revenue management is a process of controlling inventories, enabling the airline to maximize its profits. For an airline, controlling inventory equates to determining how many seats should be made available to willing purchasers for reservation, at what prices. In the short term, most of an airline's costs are fixed with very little or negligible variable costs. Thus, maximizing revenue becomes a close proxy for maximizing profit.

The concept of revenue management is not specific to the airline industry. It has been applied to numerous other businesses which have similar characteristics. These include: car rental, broadcasting, cruise lines, internet service provision, lodging, and railways. Common characteristics of yield management practice are (Kimes [21]): ability to segment markets, relatively fixed capacity, perishable inventory, product sold in advance, fluctuating demand, and low marginal sales cost/high marginal capacity change cost.

In 1992, R.L. Crandall, Chairman and CEO of AMR (American's parent company) estimated that "yield (revenue) management has generated \$1.4 billion in incremental revenue in the last three years" (Smith, Leimkuhler, and Darrow [30]). By 1998 Tom Cook, President of SABRE Decision Technologies, had increased the estimated impact to "almost \$1 billion in annual incremental revenue" (Cook [8]).

Since the early 1990s the car rental industry has also given attention towards revenue management. Most work in the area of car rental revenue management has been the application of airline revenue management methods to the car rental setting. Carroll and Grimes [7] and Geraghty and Johnson [15] provide accounts of the state-of-the-art in car rental revenue management.

One of the distinct differences of car rental revenue management from its application to airlines is the degree to which price changes. Airlines typically have several discrete price classes, across which prices are fairly constant. These price or fare classes often have restrictions on advanced booking or Saturday night stay in an effort to segment business and leisure travelers. Airlines change prices by opening or closing these different fare classes. Car rental firms also have discrete price classes for the different types of cars (economy versus luxury) but actively change the prices within these classes on a daily basis. Blair and Anderson [6] give an accurate account of pricing activity at Dollar Rent A Car. The active or dynamic price changes in car rental revenue management add a new complexity to airline revenue management. 
Weatherford and Bodily [31] and McGill and VanRyzin [23] summarize recent research in airline revenue management. Airline revenue management typically looks at allocating plane capacity across a finite set of fare classes (across which price is fairly constant). Early methods of application (Littlewood [22] and Belobaba [3]) involved newsboy-like heuristics to partition capacity across fare classes. These methods have been extended to include other elements of buyer behavior such as diversion to other fare classes (Pfeifer [27] and Belobaba and Weatherford [4]). A second stream of research has looked at the complexities resulting from hub-and-spoke networks. Early mathematical programming approaches (Glover et al. [16]) included full passenger itineraries from a deterministic standpoint, with later approaches (Williamson [32], Smith and Penn [29], and Simpson [28]) using dual prices in the allocation decision.

More closely related to car rental revenue management is the field of dynamic pricing. Typically dynamic pricing approaches assume that demand is a stochastic function of price, and that only one price is available (posted) at a given time. Gallego and VanRyzin [13] look at dynamic pricing of inventories, providing structural results for certain classes of stochastic demand. They later extend their results to network effects (Gallego and VanRyzin [14]). Feng and Gallego [11] provide a dynamic pricing approach that fits the airline model more closely. They model cases with two predetermined prices where demand follows a Poisson process. Later they extend their work to allow for multiple classes (Feng \& Xiao [12]).

We develop a new approach to dynamic pricing, one in which price itself is a random variable. Current practice assumes prices are set by firms to control demand. We illustrate that the commodity nature of the rental car business requires a more detailed approach to modeling price as an exogenous variable. 


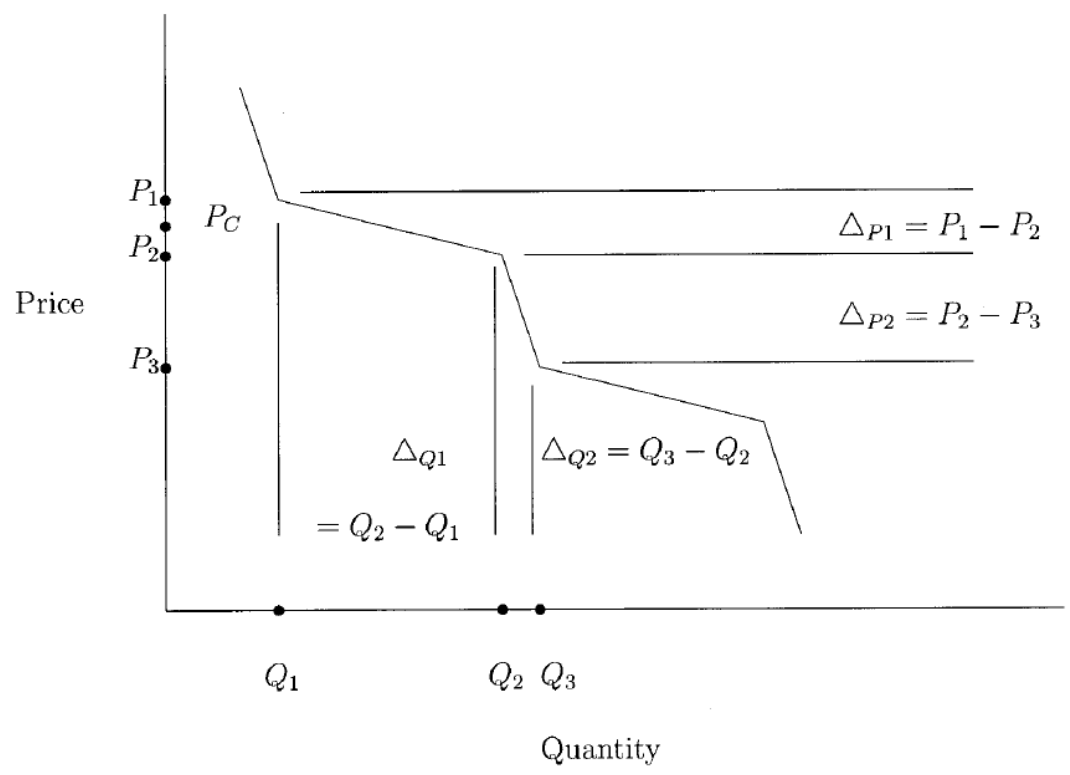

Figure 1. Car rental price elasticity.

\section{Pricing Model}

The car rental industry is not as price sensitive as the airline industry. Price changes do generate subtle changes in demand, but what is more important is one car rental firm's price against its competition's (Blair [5]). Figure 1 is a simplified illustration of price elasticity within the car rental industry. Price elasticity, the percentage change in demand per percentage change in price describes the sensitivity of sales volume to price changes. In Figure 1, demand is relatively inelastic over price changes from $P_{2}$ to $P_{3}$ as very little additional sales result ( $\Delta_{Q 2}$ is small), while very elastic from $P_{1}$ to $P_{2}\left(\Delta_{Q 1}\right.$ is quite large). Over the range $P_{1}$ to $P_{2}$ the price has crossed a competitor's price $P_{c}$, generating the volume increase (decrease) whereas from $P_{2}$ to $P_{3}$ it is still priced between two competitors, or already has the lowest price.

For simplicity, we consider a single type of business; car rental for equal duration (e.g., daily, weekly, or over the weekend) on an average daily revenue basis. All the cars are assumed identical so that at any given time the price is uniform across all cars available for rent at the same point in the future. The window over which reservations are accepted is of duration $T$, this window is typically of the order 90 days. We suppose that during the period $0 \leq t \leq T$ cars can be reserved for use on the first day after $t=0$ but that the full rental cost is paid at the time of reservation. At time $t=T$ reservations stop and no unreserved cars can be rented, representing a lost revenue opportunity. 
We will derive a model of this process. This model will be discrete in that we divide the time period into / subintervals. For example, the period of three months may be divided into 12 one week intervals, or more representative of current practice into 90, daily periods (Geraghty and Johnson [15]) allowing for daily price changes. We utilize subintervals of equal length, noting that the hypothesis of equal intervals may be relaxed at little computational cost. In addition, we will separately follow each available car, solving a subproblem for each car for each subperiod. We require a submodel for the demand for cars as a function of time.

Given the response to price as shown in Figure 1 we will model price as a random process, in essence modeling the rental car firm as a price taker, unable to arbitrarily price, rather having to price relative to competition. We will assume that the price to rent a car can be modeled by a general stochastic differential equation of the form

(1)

$$
d P=\mu(P, t) d t+b(P, t) d X
$$

where $P=P(t)$ is the price of a one day rental, $t$ is the time, and $\mu$ and $b$ are given functions of $P$ and $t$. In the PDE framework which follows we have wide flexibility to choose $\mu$ and $b$ to fit the data. The numerical solution of the differential equations will not be appreciably more difficult for most reasonable choices of $\mu$ and $b$. To be concrete in what follows, we choose

$\mu(P, t)=\alpha(L(t)-P)$,

$$
b(P, t)=\sigma P .
$$

Equation (2) states that the random process governing rental cars is mean-reverting with rate $\alpha$ to a time-dependent mean price $L(t)$. Mean reversion appears a reasonable model as, while rental car prices fluctuate from day to day, they seem to be bounded both below and above. The lower bound exists because prices cannot stay significantly below the marginal cost of renting the car for too long or the rental operator would have to exit the market. The upper bound exists both because of the competitive, winner take all, nature of the rental car market and because of the price elasticity of consumers. Both of these effects support a mean reverting price model. We choose (3) with constant volatility parameter $\sigma$ to represent a constant relative fluctuation in the prices, $\sigma$ may be constant or a function of time without any change in formulation. This price process is really for the price of a 
reservation, the price price paid in the future for a reservation today. The model is then similar to a forward price for a commodity, which are also often modelled as mean reverting.

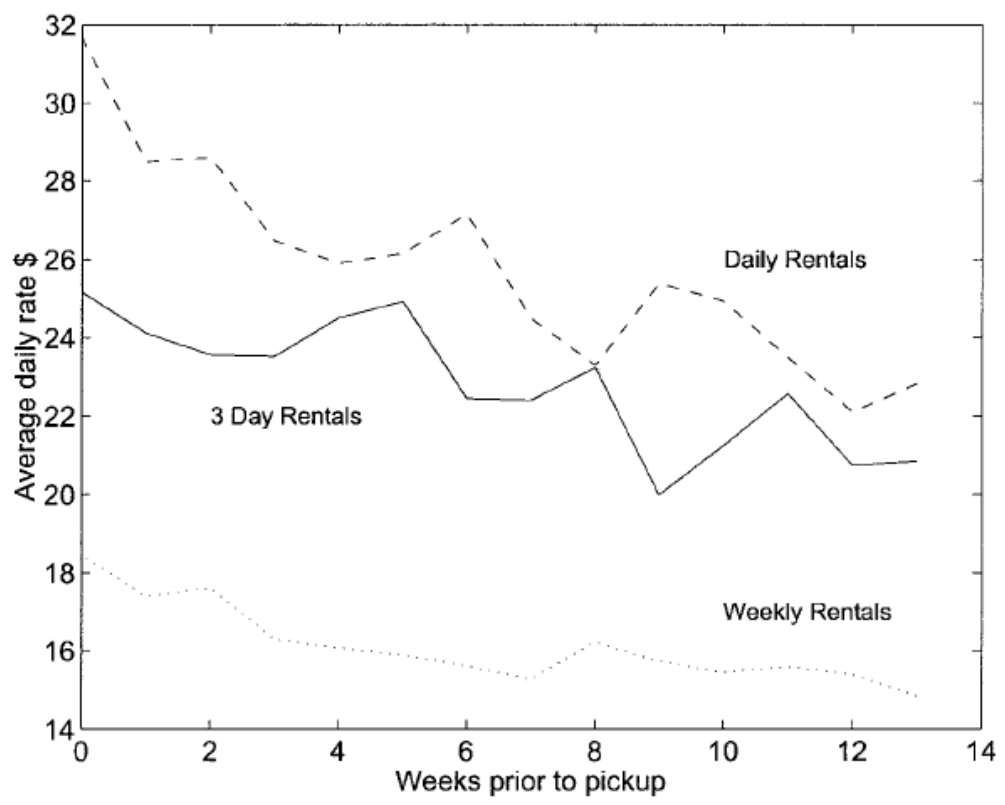

Figure 2. Average daily rental rates—weekday pickup for Denver economy cars.

The only choice which remains is how to represent the mean rental car price as a function of days before rental. We choose a simple linear increasing function of time for this. To motivate this choice, we examine Figure 2 which plots the average realized price per day of single day, 3-day, and week-long car rentals for Dollar Rent A Car against weeks before pickup. These data, provided by Dollar, are for economy cars for weekday pickup rented at Denver International airport during calendar year 2001. In every case a subtle but noticeable upward trend in the average rental price is observed. In any event, the large fluctuations in the dataset we present make it difficult to justify a more complicated model for the mean.

Consider a time interval $0 \leq t \leq T$ such that $t=0$ corresponds to the start of the reservation time and $t=T$ to the time of rental. We suppose that we have $M$ cars to rent and that they can be rented at a daily varying price given by the stochastic differential equation (1). The number of cars available at the start of the booking cycle, $t=0$, will be both a function of fleet size and how that fleet is allocated across different lengths-of-rent, typically determined using linear programming (Geraghty and Johnson [15]). Linear programming is used to maximize expected revenue by allocating cars to the different lengths-of-rent, while constraining the allocation such that allocated cars (by length-of-rent class) is no more than expected demand, and total cars allocated no more than available fleet. We 
suppose that cars can only be rented at fixed intervals given by $0<t_{1}<t_{2}<\ldots t_{N}<T$, where $t_{j}=j^{*} \Delta t$. The total number of cars that can be rented in a period (the demand) is a given function of price $F(P)$. For period $j$, for generality, we suppose both additive forms of demand

$F\left(P_{j}\right)=\beta_{0}+\beta_{1} * P_{j}$

and multiplicative

$$
F\left(P_{j}\right)=\beta_{0} * P_{j}^{\beta_{1}}
$$

with coefficients $\beta_{0}$ and $\beta_{1}$ constant or indexed with time.

Write the value of the cash flow for the remaining cars as

$V_{m}^{j}$

at the beginning of a time interval $j$, where $m$ cars have been rented. We now consider the interval $t_{j} \leq$ $t \leq t_{j+1}$ where we will be renting cars at $t=t_{j+1}$. The car rental firm is faced with the decision to accept a reservation or wait for potential future (higher) revenues. The firm has the option of renting or holding the car.

\begin{tabular}{l|l|l|l|l|l|l|}
$m \backslash j$ & 0 & 1 & 2 & 3 & 4 \\
\hline 0 & & & & & \\
\hline 1 & & & & & \\
\hline 2 & & & & & \\
\hline 3 & & & & & \\
\hline \multicolumn{7}{|c|}{$t_{1}$} \\
\hline
\end{tabular}

Figure 3. Subproblem layout.

Since the price is given by a stochastic differential equation, the cash value $V_{m}^{j}$ can be considered as a European option starting at $t=t_{j}$ with a payout of $\phi$ at $t=t_{j+1}$. It is a European option as we are assuming it can be exercised only at time $t=t_{j+1}$. If we rent a car at $t_{j+1}$, the expected cash flow is given by

$$
\phi=V_{m+1}^{j+1}+P_{j+1}
$$

where $P_{j+1}$ is the rental price we get when the car is picked up (rented) and $V_{m+1}^{j+1}$ is the expected future cash flow from the remaining cars. In general, if we rent $k$ cars, the payout is

$\phi=V_{m+k}^{j+1}+k P_{j+1}$, 
where $m+k \leq M$; i.e., we cannot rent more cars than we have available. If we do not rent any cars, the payout is just the cash flow at $t_{j+1}$, i.e., $V_{m}^{j+1} V m j \_1$. Thus the complete payout at $t_{j+1}$ is

$$
\phi=\max \left(V_{m}^{j+1}, V_{m+1}^{j+1}+P_{j+1}, V_{m+2}^{j+1}+2 P_{j+1}, \ldots, V_{m+k}^{j+1}+k P_{j+1}\right),
$$

with $m+k \leq M$ and $k \leq F\left(P_{j+1}\right)$; i.e., we cannot rent more cars than we have, and the number we rent, $k$, is limited by demand at price $P_{j+1}, k \leq F\left(P_{j+1}\right)$.

For the final period $j=N$, the payout is just the number of cars that can still be rented out; i.e.,

$$
\phi=k P_{N},
$$

where $m+k \leq M$ and $k \leq F\left(P_{N}\right)$.

We can now represent the problem as a collection of subproblems; this is shown on Figure 3 as a grid with $0 \leq j \leq 4$ and $0 \leq m \leq 3$. For each cell in the grid we can derive a Black-Scholes-like partial differential equation. We can use the payout at the right-hand side of the cell to calculate the value at the left-hand side. This standard derivation may be found for instance in Dixit and Pindyck [10]. To illustrate this, consider the cell that corresponds to $j=3$ and $m=1$. Here the payout is

(5)

$$
\phi=\max \left(V_{1}^{4}, V_{2}^{4}+P_{4}, V_{3}^{4}+2 P_{4}\right) .
$$

We use this as the initial condition for an equation for $V_{1}^{3}$.

Now consider a general cell located between $t_{j} \leq t \leq t_{j+1}$ and $0 \leq m \leq M$. Using the pricing model (1) we can develop the governing partial differential equation for the cash flow $V(P, t)$.

Starting with a model for rental prices $P$,

$d P=\mu(P, t) d t+b(P, t) d X$,

the goal is to determine the value of the option to rent the car at time $t$ given that if it is not rented at time $T$ a revenue opportunity is lost, this option value is $V(P, t ; T)$.

If this were a financial option we could establish a hedging argument to price this in a riskneutral measure. This argument may be expressed in PDE form by a simple application of Bellman's principle using a risk-free discount rate $r$ (Dixit and Pindyck [10]) 
(6)

$$
\frac{\partial V}{\partial t}+\frac{1}{2} b^{2} \frac{\partial^{2} V}{\partial P^{2}}+\mu \frac{\partial V}{\partial P}-r V=0 .
$$

However, unlike financial options, it is less clear that risk can be hedged away in this setting. Perhaps something could be done along the lines of hedging midsize cars against luxury cars, but there are practical difficulties with this idea. We can, however incorporate a risk-adjustment as follows. We use a Sharpe ratio $\lambda=(\mu-r) / \sigma$ to denote the riskiness of the investment, we derive a PDE using Bellman's principle. Thus pricing in a risk-neutral measure corresponds to $\lambda=0$, and recovers the earlier PDE [Eq. (6)]

(7)

$$
\begin{aligned}
& \frac{\partial V}{\partial t}+\frac{1}{2} b^{2} \frac{\partial^{2} V}{\partial P^{2}}+(\mu-\lambda b) \frac{\partial V}{\partial P}-r V=0 . \\
& \text { In our model of rental car prices } b=\sigma P . \text { Now, }
\end{aligned}
$$

$$
\frac{\partial V}{\partial t}+\frac{1}{2} \sigma^{2} P^{2} \frac{\partial^{2} V}{\partial P^{2}}+(\mu-\lambda \sigma P) \frac{\partial V}{\partial P}-r V=0,
$$

where $\lambda$ is the market price of risk.

If we set $\lambda=0$, then the solution follows that of a risk-neutral or expected value maximizing decision-maker. Note that our numerical results indicate that the solution is quite insensitive to the choice of $\lambda$. With $\lambda=0$ and Eq. (2) we have $\mu=\alpha(L(t))-P)$. We will replace the time variable $t$ with a local time variable $\tau$ within the rectangle which starts at $t_{j+1}$ and ends at $t_{j}$. We also replace the variable $t$ with $t_{j+1}-\tau$ in order to get a forward problem. Thus the governing partial differential equation becomes

$$
\frac{\partial V}{\partial \tau}=\frac{1}{2} \sigma^{2} P^{2} \frac{\partial^{2} V}{\partial P^{2}}+\alpha(L-P) \frac{\partial V}{\partial P}-r V
$$

Here $V_{m}(P, j \Delta t)=v_{m}^{j}(P)$ and for convenience $L(t)$ is expressed as $L$.

The initial condition for this equation which is obtained from (4) is

$$
V(P, 0)=\max \left(V_{m}^{j+1}, V_{m+1}^{j+1}+P_{j+1}, V_{m+2}^{j+1}+2 P_{j+1}, \ldots, V_{m+k}^{j+1}+k P_{j+1}\right),
$$


with $m+k \leq M$ and $k \leq F\left(P_{j+1}\right)$.

We also require boundary conditions. The situation at $P=0$ is quite interesting and the analysis outlined below is similar to that treated by d'Halluin, Forsyth, and Labahn [9] in their discussion of the single factor interest rate model.

If we let $P \rightarrow 0$, Eq. (9) becomes

$$
\frac{\partial V}{\partial t}=\alpha L \frac{\partial V}{\partial P}-r V
$$

Since $\alpha L>0$, this is a hyperbolic equation with outgoing characteristic, so we can expect that no boundary condition is required at $P=0$. However, for finite but small values of $P$, Eq. (9) has a diffusive term and the question is whether this term tends to zero sufficiently rapidly that no boundary condition is required. If it does not, we require a boundary condition. This question has been answered by Oleinik and Radkevic [26], who showed that, for any equation of the form

$$
\frac{\partial \phi}{\partial t}=a(x) \frac{\partial^{2} \phi}{\partial x^{2}}+b(x) \frac{\partial \phi}{\partial x}+c(x) \phi
$$

no boundary condition is required at $x=0$ if

$$
\lim _{x \rightarrow 0}\left(b(x)-a^{\prime}(x)\right) \geq 0 .
$$

For (9) this condition is satisfied provided $\alpha L>0$ which is always the case.

Thus we see that no boundary condition is required at $P=0$ and we will obtain a finite difference equation there by discretizing (11).

For large values of $P$ it is clear that $V$ will depend linearly on $P$, so we will use

$$
\frac{\partial^{2} V}{\partial P^{2}} \rightarrow 0 \quad \text { as } \quad P \rightarrow \infty
$$

For the last time interval we can write down an exact solution of the form

$$
V_{m}^{N}(\tau)=A(\tau)+B(\tau) P
$$

since the initial condition is $V_{m}^{N}(0)=K P$, where $K$ is a given constant.

The next section details numerical solution procedures for the above model. 


\section{Numerical Procedure}

In general, the mathematical model developed above cannot be solved analytically. To generate pricing recommendations, we employ a numerical approach. We use a standard implicit procedure with $P_{j}=j \Delta P$ and $V_{j}^{n}=V(j \Delta P, n \Delta \tau)$ for $j=0, \ldots, J$ and $n=0, \ldots, n t$. Here $I \Delta P=P_{\text {max }}$ and $n \Delta \tau=\Delta T$, where $P=P_{\max }$ is the price at which we impose the boundary condition $V_{P P}=0, \Delta T$ is the length of each period, and $I$ is an identity matrix. If we use central difference formulae, the resulting difference equations can be written in the form

$$
(I+\theta \Delta t A) V^{n+1}=(I-(1-\theta) \Delta t A) V^{n}
$$

for $0<j<J$, where

$$
A=\left(\begin{array}{ccccc}
r+b_{0} & -b_{0} & & & \\
-a_{1} & r+a_{1}+b_{1} & -b_{1} & & \\
& & -a_{J-1} & r+a_{J-1}+b_{J-1} & -b_{J-1} \\
& & & a_{J} & r+a_{J}
\end{array}\right)
$$

For $j=1, \ldots, J-1$,

$$
a_{j}=\frac{\sigma^{2} P_{j}^{2}}{2 \Delta P^{2}}-\frac{\alpha\left(L-P_{j}\right)}{2 \Delta P},
$$

$b_{j}=\frac{\sigma^{2} P_{j}^{2}}{2 \Delta P^{2}}+\frac{\alpha\left(L-P_{j}\right)}{2 \Delta P}$,

$$
b_{0}=\frac{\alpha L}{\Delta P},
$$

$$
a_{j}=\frac{\alpha\left(L-P_{j}\right)}{\Delta P} .
$$


It is possible for $a_{j}$ to be negative for small values of $j$, i.e., near $P=0$; this corresponds to the governing equation being convection-dominated. In this case the system of difference equations are no longer diagonally dominant, and we can expect their solution to oscillate. In order to circumvent this problem, we will use upstream weighting for the convective term and get

$$
a_{j}=\frac{\sigma^{2} P_{j}^{2}}{2 \Delta P^{2}},
$$

$$
b_{j}=\frac{\sigma^{2} P_{j}^{2}}{2 \Delta P^{2}}+\frac{\alpha\left(L-P_{j}\right)}{\Delta P} .
$$

This approximation is only first order, but since it will only be used away from the region of interest, this should not cause any problems.

We define $\theta$ in (13) to be a parameter between 0 and 1 . Setting $\theta=1 / 2$ gives the standard CrankNicolson procedure, which is second order in both $\Delta t$ and $\Delta P$, while $\theta=1$ produces a fully implicit procedure. While it is well known that in certain cases the Crank-Nicolson procedure has a tendency to produce solutions with unphysical oscillations, this seems not to be the case for this problem so we have mainly used $\theta=1 / 2$.

$$
\text { At } P=0 \text {, i.e., } j=0 \text {, we will discretize Eq. (11) using an upstream weighting for the diffusive term. }
$$

Hence we have

$$
\begin{gathered}
a_{0}=0, \\
b_{0}=\frac{\alpha L}{\Delta P} .
\end{gathered}
$$

For large values of $P$ we truncate the region at $P=P_{\max }$, and there we appeal to boundary condition (12) to set the second derivative to zero. Then a standard central difference approximation gives

$$
V_{J+1}=2 V_{J}-V_{J-1}
$$

Yielding 
$a_{J}=-\frac{\alpha\left(L-P_{J}\right)}{\Delta P}$

The set of difference equations (13) is tridiagonal so a very fast form of Gaussian elimination can be applied. For details see Morris [25].

\section{Model Validation}

As mentioned in Section 4 our model does not typically admit exact solutions. However, for special cases we can derive an exact solution. We can use these different cases to validate our numerical approach.

\section{Analytic Solution}

Let us first derive an expression for the mean as a function of time for our pricing model. In order to simplify the analysis, we will only consider the case where the mean of the price equals a constant, $m$. Thus the pricing model (1), (2), and (3) becomes

$d P=\alpha(m-P) d t+\sigma P d X$,

with initial condition $P(0)=P_{0}$.

It is well known that the mean of this process follows the path

$\bar{P}(t)=P_{0} \exp (-\alpha t)+m(1-\exp (-\alpha t))$.

We now use this result to derive an exact solution for the situation in which we are in an unconstrained environment in which we can rent $k$ cars per period for $N$ periods, each spaced by time $\Delta T$. The initial price of the cars is $P_{0}$. There is no optionality here so the present value of the expected value of the cash flows arising from the business is: 
(22)

$$
\begin{aligned}
V= & k \bar{P}(0)+k \bar{P}(\Delta T) \exp (-r \Delta T)+\cdots+k \bar{P}((N-1) \Delta T) \exp (-r(N-1) \Delta T) \\
= & k \sum_{j=0}^{N-1} \exp (-j r \Delta T)\left[P_{0} \exp (-j \alpha \Delta T)+1-\exp (-j \alpha \Delta T)\right]=k\left(P_{0}-m\right) \sum_{j=0}^{N-1} \exp [-j(r+\alpha) \Delta T] \\
& +k m \sum_{j=0}^{N-1} \exp (-j r \Delta T)=k\left(P_{0}-m\right) \frac{1-\exp [-N(r+\alpha) \Delta T]}{1-\exp [-(r+\alpha) \Delta T]}+k m \frac{1-\exp (-N r \Delta T)}{1-\exp (-r \Delta T)} .
\end{aligned}
$$

If $V$ is plotted against $P_{0}$, the solution is seen to be a straight line.

\section{$\underline{\text { Validation }}$}

In order to validate our numerical approach, we look at situations in which the car rental problem can be solved exactly in the form above. Two such cases follow.

- There is a surplus of cars and the price is large compared with the mean.

- Small volatility and small values of $P$

\subsubsection{Case 1}

Let us consider the case where there is a surplus of cars available so that the full number of cars, say $K$, can be reserved in every period. In addition we write the expression for the mean price in the form

$$
L=a+b \tau
$$

where $a$ and $b$ are positive constants. Then the governing equation for each period is

$$
V_{\tau}=\frac{1}{2} \sigma^{2} P^{2} \frac{\partial^{2} V}{\partial P^{2}}+\alpha(a+b \tau-P) \frac{\partial V}{\partial P}-r V
$$

where $\tau=0$ is the beginning of the period and $\tau=\Delta T$ is the end of the period.

In the last period we denote the value of the cash flow as $V_{N}(P, \tau)$, and it is clear that the initial condition is $V_{N}(P, 0)=K P$, so the solution can be written in the form

$$
V_{N}(P, \tau)=A_{N}+B_{N} P
$$

We substitute this into Eq. (22) for $V$ to see

$$
A_{N}=-K\left(a+\frac{b}{\alpha}+b t\right) e^{-(\alpha+r) \tau}+K\left(a+\frac{b}{\alpha}\right) e^{-r \tau}
$$


and

$$
B_{N}=K e^{-(\alpha+r) \tau} .
$$

Table 1. Validation Case 1-excess supply.

\begin{tabular}{lllll}
\hline & $V(P, 0)$ & $V(P, 0)$ & $V(P, 0)$ & $V(P, 0)$ \\
$P$ & $N=3$ & $N=6$ & $N=12$ & Analytic \\
\hline 1 & 20.5621 & 22.1741 & 24.7186 & 20.0000 \\
2 & 32.3604 & 32.6211 & 33.2656 & 32.3018 \\
3 & 44.6130 & 44.6079 & 44.8372 & 44.6036 \\
4 & 56.9067 & 56.9166 & 56.9557 & 56.9054 \\
\hline
\end{tabular}

In the $N$ - 1 period the cash flow is $V_{N-1}$ with initial condition $A_{N}(\tau)+B_{N}(\tau) P+K P$, so, using the same procedure as above, we see that the solution can be written in the form

$V_{N-1}(P, t)=A_{N-1}+B_{N-1} P$.

Here

$$
A_{N-1}=-\left(B_{N}+K\right)\left(a+\frac{b}{\alpha}+b \tau\right) e^{-(\alpha+r) \tau}+\left(B_{N}+K\right)\left(a+\frac{b}{\alpha}\right) e^{-r \tau}
$$

and

$$
B_{N}=\left(B_{N}+K\right) e^{-(\alpha+r) \tau} .
$$

The subsequent solutions all have the same form.

This is, of course, the same solution we derived in subsection 5.1 using the mean of the pricing process.

Now consider the case where $\sigma=1, T=0.0833, \alpha=4$, the total number of cars that can be rented out over one period is 10 and the total number of cars available is 20 . Thus it is clear that if the total number of periods is greater than 2 and if the price at which cars can be rented out is considerably greater than 1, all the cars will be rented out in the first two periods, and the exact solution derived above will be applicable.

Here Table 1 indicates that as price increases from a low of 1 to a high of 4 , the numerical solution approaches the analytical. Similarly, as the number of periods in the numerical solution approaches that of the analytical solution (2), then the results converge. As expected, as the number of periods in the numerical solution increases, more opportunities for price increases are available, and the numerical result will diverge from the analytical. These results are less pronounced as prices increase. 
Case 2

The mean reverting pricing model that we are using is not easy to solve. However, if we assume that the volatility is small compared to the mean reversion term, we get, as in subsection 5.1 with units selected such that $m=1$,

$$
P(t)=1-\left(1-P_{0}\right) e^{-\alpha t},
$$

where $P_{0}=P(0)$ and $P(t)$ should now be interpreted as the mean. If we apply this to the case where there is 3 periods, each of length $1 / 12$ years, we see that

$$
\begin{gathered}
P_{1}=1-e^{-\alpha / 12}, \\
P_{2}=1-\left(1-P_{1}\right) e^{-\alpha / 12}, \\
P_{3}=1-\left(1-P_{2}\right) e^{-\alpha / 12},
\end{gathered}
$$

where $P_{0}=0$. For $\alpha=4$ we get that

$$
P_{1}=.283, \quad P_{2}=.48, \quad P_{3}=.632 \text {. }
$$

Thus for the case where, as above, we can rent out 10 cars per period but only a total of 20 cars, an estimate of the cash flow when $r=0$ is $10 P_{2}+10 P_{3}$, which equals 11.18 . The numerical procedure generates the same result. We can proceed in this manner, and for $N=6$ we find that an approximate value for the cash flow at $t=0$ is 15.47 while the numerical result is 16.89 . As $N$ becomes larger, it is clear that the expected value of $P$ tends to 1 so the value of the cash flow should tend to 20 . However, the numerical result for $N=12$ is 21.74 .

In general, these results can be interpreted as at least partial validation of the numerical procedure.

\section{Numerical Example}

We require representative values of the different parameters that appear in the model. Two of these, which originate in the pricing model, are not easy to estimate with any degree of accuracy. We will show how to get reasonable values of the parameters and then show that our computational model is not overly sensitive to reasonable changes in them.

Let us first consider the volatility $\sigma$. If we consider the pricing with $P$ being close to the mean $L$, we get approximately that 


$$
d P=\sigma d X,
$$

where $X$ is a Wiener process; one can think of $d X$ as being a random variable, drawn from a normal distribution with mean zero and variance $d t$. Since $d X$ scales as $\sqrt{d t}$ we see that an estimate for $\sigma$ is given by

$$
\sigma=\frac{d P}{\sqrt{d} t}
$$

If we suppose that the change in the price of rental cars over a 1 week period is $10 \%$, and we estimate $\sqrt{52}=7$, this implies that _ should be of the order $70 \%$ per year.

In order to get an estimate for $\alpha$, the rate at which the car rental price reverts to its mean, let us consider the pricing model with no stochastic noise. Then

$$
d P=-\alpha(P-L) d t
$$

where we will assume that the mean price $L$ is constant. This equation can be integrated to get

$$
P=L+\left(P_{0}-L\right) e^{-\alpha t},
$$

where $P_{0}=P(0)$. Approximate $e^{-\alpha t}$ by 1 - $\alpha t$ so

$$
P=L+\left(P_{0}-L\right)(1-\alpha t)
$$

Thus we see that the time $t^{*}$ that it takes the price to return to the mean is given by $1-\alpha t^{*}=0$. The data indicates $t^{*}$ is usually of the order of two days so $\alpha$ is approximately $180 /$ year.

Table 2. Parameter values-numerical example.

\begin{tabular}{lc}
\hline \multicolumn{1}{c}{ Parameter } & Estimate \\
\hline Number of periods $N$ & 12 \\
Length of each period & 1 week \\
Total number of cars & 50 \\
Discount rate $r$ & $5 \%$ per year \\
Average minimum rental price & $25 \$$ \\
Average maximum rental price & $30 \$$ \\
Demand at zero price & 20 cars \\
Slope of demand curve & $-\frac{1}{15}$ \\
Volatility $\sigma$ & 1 \\
Return rate $\alpha$ & 180 \\
\hline
\end{tabular}


The remaining parameters are easily found and we will use the set of parameter values given in Table 2.

Using these parameters values, we generate the plots shown in Figure 4 which shows the number of cars that should be rented out for different prices at time $t=0$, i.e., at the beginning of the first of 12 rental periods of a week each, in order for the franchise to maximize the total cash flow. The price model underlying the figure is relatively flat with an average price at time zero of $\$ 25$ rising to a maximum (average) of $\$ 30$ at the end of the reservation period. Price volatility is moderate, representing about a $12 \%$ change on a weekly basis $(\sigma=1)$ with quick price reversion, $\alpha=180$. Base demand is 20 units with elasticity $-1 / 15$. The three series plotted in Figure 4 are for three different levels of risk adjustment. The series with $\lambda=0$ is equivalent to a risk neutral or expected value approach, whereas $\lambda=5$ and $\lambda=10$ represent increasing value in risk. The increased value placed on risk means the options are worth more to the firm resulting in them holding on to the options longer, requiring larger rates to rent the cars.

It is important to note that Figure 4 is a merging of supply and demand. At lower prices the car rental firm is limiting supply, not making cars available till prices exceed certain limits. At higher prices, the firm is willing to rent but sales are capped by a lack of demand.

The interesting part is that even if the mean price is $\$ 25$ per car, the risk neutral franchise should not rent cars out for less than about $\$ 23$ a car. This confirms earlier results where Anderson and Blair [2] indicated that early rentals at deeply discounted rates are costly, from a revenue standpoint, to the car rental firm, indicating that perhaps car rental firms are discounting too deeply early in the booking cycle.

Let us now consider the sensitivity of our results to changes in $\sigma$ and $\alpha$. If we change $\sigma$ from 0.1 to 2.0 , we see that the minimum rental price changes from $\$ 22.60$ to $\$ 22.80$. Similarly, if we change $\alpha$ but keep the other parameters at the values given in Table 2, we get the results in Table 3. Table 3 shows that the slower prices tend to revert, the more likely a firm is to rent early at deeply discounted rates.

The procedure is implemented in $\mathrm{C}^{++}$in about 600 lines of code. Execution time for a problem with 12 periods is about five seconds on a standard PC (Pentium III, $550 \mathrm{MHz}$ ). Since the routine must be run once per period the total execution time will be around 35 seconds. 


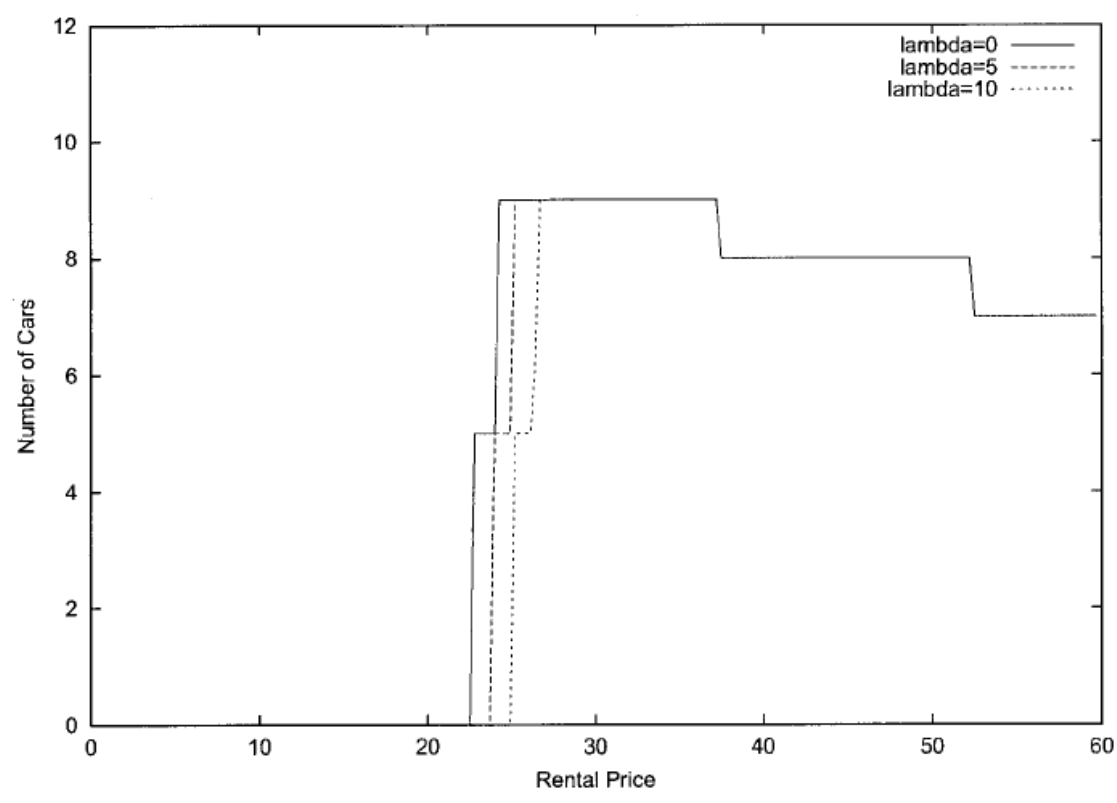

Figure 4. Rates and availability at initial booking.

\section{Discussion}

We have derived a new model for revenue management and applied it to car rentals. This model is based on the concepts of real option theory and is related to the swing options used in the power industries. We have derived some exact results and used these in the validation of a computational model of the process.

The method provides an approach to including competitive effects in revenue management settings. For commodity based service businesses, like car rentals (where customer switching costs are low), a firm's pricing decisions may be impacted by their price relative to competition. Our approach to modeling price as a stochastic differential equation is a novel approach to including these competitive effects in revenue management applications.

Table 3. Sensitivity analysis-rate of reversion to mean.

\begin{tabular}{rc}
\hline \multicolumn{1}{c}{$\alpha$} & First rental price \\
\hline 2 & 26.0 \\
5 & 25.5 \\
10 & 24.0 \\
100 & 22.8 \\
180 & 22.8 \\
\hline
\end{tabular}


We suppose that the management of the business has a database which allows calibration of stochastic models of rental price development. At present our approach does not include impacts of inventory sharing across multiple rental periods, i.e., a car may be rented overnight or for 3 days. Similarly our model does not include effects created by multiple car classes, i.e., the ability to rent a higher valued car to a customer desiring a lower valued car. These limitations may be relaxed by jointly modeling the price process for different lengths of rent for different classes of cars. These price processes would need to be correlated as prices would tend to move together across classes and rental lengths.

While we have illustrated the application of Real Options to revenue management using car rentals as an example, the general approach is extendible to any industry with active price changes. Intense competition only helps to motivate the application of our methods.

\section{Acknowledgements}

The authors wish to thank Montgomery Blair of Dollar Thrifty Automotive Group, for provision of data and insight into car rental revenue management. Financial support for this research was received from Natural Sciences and Engineering Research Council of Canada (NSERC) and Mathematics of Information Technology and Complex Systems (MITACS).

\section{References}

[1] M. Amram and N. Kulatilaka, Real options: Managing strategic investment in an uncertain world, Harvard Business School Press, Boston, 1999.

[2] C. Anderson and M. Blair, Performance monitor: The opportunity costs of revenue management, J Revenue Pricing Management 2(4) (2004), 353-367.

[3] P. Belobaba, Air travel demand and airline seat inventory management, Unpublished doctoral dissertation, Flight Transportation Research Laboratory, MIT, Cambridge, MA, 1987.

[4] P. Belobaba and L. Weatherford, Comparing decision rules that incorporate customer diversion in perishable asset revenue management situations, Decision Sci 27 (1996), 3-43.

[5] M. Blair, Dynamic pricing; the new diversity of revenue management, IFORS Triennial Meeting, Edinburgh, Scotland, 2002.

[6] M. Blair and C. Anderson, Performance monitor, J Revenue Pricing Management 1(1) (2002), 57-66. 
[7] W. Carroll and R. Grimes, Evolutionary changes in product management: experiences in the car rental industry, Interfaces 25(5), (1995), 84-104.

[8] T. Cook, Sabre soars, OR/MS Today 25(3), (1998), 26-31.

[9] K.V. d'Halluin, P.A. Forsyth, and G. Labahn, Numerical pde approach for pricing callable bonds, working paper, Department of Computer Science, University of Waterloo, Waterloo, Ontario, Canada, 2001.

[10] A.K. Dixit and R.S. Pindyck, Investment under uncertainty, Princeton University Press, Princeton, NJ, 1994.

[11] Y. Feng and G. Gallego, Optimal starting times for end-of-season sales and optimal stopping times for promotional fares, Management Sci 41 (1995), 1371-1391.

[12] Y. Feng and B. Xiao, Optimal policies of yield management with multiple predetermined prices, Oper Res 48 (2000), 332-343.

[13] G. Gallego and G. VanRyzin, Optimal dynamic pricing of inventories and stochastic demand over finite horizons, Management Sci 40 (1994), 999-1020.

[14] G. Gallego and G. VanRyzin, A multi-product dynamic pricing problem and its applications to network yield management, Oper Res 45 (1997), 24-41.

[15] M. Geraghty and E. Johnson, Revenue management saves National Car Rental, Interfaces 27(1) (1997), 107-127.

[16] F. Glover, R. Glover, J. Lorenzo, and C. McMillan, The passenger mix problem in the scheduled airlines, Interfaces 12 (1982), 73-79.

[17] F. Harris and P. Peacock, Hold my place, please, Market Management 4 (1995), 34-44.

[18] J.C. Hull, Options, futures and other derivatives (5 edition), Prentice Hall, Upper Saddle River, NJ, 2003.

[19] P. Jaillet, E. Ronn, and S. Tompaidis, Valuation of commodity-based swing options, working paper, McCombs School of Business, University of Texas at Austin, 2001.

[20] J. Keppo, Pricing of electricity swing options, working paper, Department of Industrial and Operations Engineering, University of Michigan, 2002.

[21] S. Kimes, Yield management: A tool for capacity constrained service firms, J Oper Management 8 (1989), 348-363.

[22] K. Littlewood, Forecasting and control of passenger bookings, AGIFORS Symp Proc, Nathanya, Israel, December 1972, pp. 95-128. 
[23] J. McGill and G. VanRyzin, Revenue management: Research overview and prospects, Transportation Sci 33(2) (1999), 233-256.

[24] R.C. Merton, Continuous-time finance, Blackwell, Cambridge, MA, 1990.

[25] J. Morris, Computational methods in elementary numerical analysis, Wiley, New York, 1983.

[26] A. Oleinik and E. Radkevic, Second order equations with nonnegative characteristic form, American Mathematical Society, Providence, RI, 1973.

[27] P. Pfeifer, The airline discount fare allocation problem, Decision Sci 20 (1989), 149-157.

[28] R. Simpson, Setting optimal booking levels for flight segments with multi-class, multi-market traffic, AGIFORS Symp Proc 25, Hikone, Japan, 1985, pp. 263-279.

[29] B. Smith and C. Penn, Analysis of alternative origin-destination control strategies, AGIFORS Symp Proc 28, New Seabury, MA, 1988, pp. 123-144.

[30] B. Smith, J. Leimkuhler, and R. Darrow, Yield management at American Airlines, Interfaces 22 (1992), 8-31.

[31] L. Weatherford and S. Bodily, A taxonomy and research overview of perishable-asset revenue management: Yield management, overbooking and pricing, Oper Res 40 (1992), 831-844.

[32] E. Williamson, Airline network seat inventory control: Methodologies and revenue impact, Unpublished doctoral dissertation, Flight Transportation Laboratory, MIT, Cambridge, MA, 1992.

[33] P. Wilmott, Paul Wilmott on quantitative finance, Wiley, Chichester, UK, 2000.

Anderson, Davison, and Rasmussen: Revenue Management: A Real Options Approach 703 